\title{
Infliximab treatment reduces depressive symptoms in patients with ankylosing spondylitis: an ancillary study to a randomized controlled trial (ASSERT)
}

\author{
Casper Webers ${ }^{1,2^{*}}$ (D) Carmen Stolwijk ${ }^{3}$, Olga Schiepers ${ }^{4}$, Thea Schoonbrood ${ }^{1}$, Astrid van Tubergen ${ }^{1,2}$,
} Robert Landewé $e^{5,6}$, Désirée van der Heijde ${ }^{7}$ and Annelies Boonen ${ }^{1,2}$

\begin{abstract}
Background: Patients with ankylosing spondylitis (AS) are at increased risk of depression. This increased risk has been hypothesized to be solely secondary due to AS-related symptoms, or additionally due to a common inflammatory pathway. From a clinical perspective, it is important to know whether treatment with tumor necrosis factor alpha inhibitors reduces depressive symptoms, while from a pathophysiological point of view, it would be insightful to understand whether such an effect would be a direct result of reduced inflammation, the result of reduced AS-related symptoms, or both. The objective of this study was to evaluate the effect of infliximab on depressive symptoms in patients with AS in a randomized-controlled trial setting.

Methods: Data were retrieved from a subgroup of patients from the AS Study for the Evaluation of Recombinant Infliximab Therapy (ASSERT). Patients were randomly allocated to infliximab $(n=16)$ or placebo $(n=7)$ until week 24 , after which all received infliximab until week 54. Associations between treatment group and depressive symptoms, measured with the Center for Epidemiological Studies Depression scale (CES-D, range 0-60 (best-worst)) at baseline and over time, were explored with generalized estimating equations (GEE).

Results: Mean CES-D score at baseline was 15.5 (SD 9.3) in the infliximab group and 17.3 (SD 5.7) in the placebo group. Twelve patients (52\%) had a CES-D score $\geq 16$, suggestive for clinical depression. After 24 weeks, mean CESD had decreased to 9.5 (SD 11.4) in the infliximab group, but was 18.0 (SD 6.9) in the placebo group. GEE revealed larger improvements in depressive symptoms $(B=-6.63,95 \% \mathrm{Cl}-13.35$ to 0.09$)$ and odds of possible depression $(\mathrm{OR}=0.02,95 \% \mathrm{Cl} 0.00$ to 0.72$)$ in the infliximab group, compared to the placebo group. Both associations largely disappeared when adjusted for self-reported disease activity and/or physical function. Additional adjustment for Creactive protein (CRP) did not change results.

(Continued on next page)
\end{abstract}

\footnotetext{
* Correspondence: casper.webers@mumc.nl

'Department of Internal Medicine, Division of Rheumatology, Maastricht University Medical Centre, PO Box 5800, Maastricht 6202 AZ, The Netherlands

${ }^{2}$ Care and Public Health Research Institute (CAPHRI), Maastricht University, Maastricht, the Netherlands

Full list of author information is available at the end of the article
} 
(Continued from previous page)

Conclusions: Depressive symptoms are common in patients with AS and active disease. Infliximab improves these depressive symptoms in AS when compared to placebo by improving disease symptoms. We did not find an indication for a direct link between CRP-mediated inflammation and depressive symptoms.

Trial registration: Trial registration (ASSERT): NCT00207701. Registered on September 21, 2005 (retrospectively registered).

Keywords: Ankylosing spondylitis, Depressive symptoms, Anti-TNF-a therapy, Randomized controlled trial

\section{Background}

Ankylosing spondylitis (AS) affects not only the patients' physical health, but also their mental well-being. A systematic review showed that patients with AS have an increased prevalence of depressive symptoms, with rates ranging between 11 and 64\% [1]. Comorbid depression in AS impacts the individual and has societal relevance, as it has been associated with work disability in inflammatory arthritis [2].

Different possible pathways might explain the increased prevalence of depressive symptoms in AS. Depressive symptoms could be secondary to disease-related impairments such as pain and limitations in physical functioning, or to psychological consequences such as worrying about the future [3]. But AS and depressive symptoms may also share a common pathophysiological pathway as both may be the result of an auto-inflammatory biological process; proinflammatory biomarkers, such as C-reactive protein (CRP) and tumor necrosis factor (TNF)- $\alpha$, have been found to be higher in depressed individuals compared to non-depressed individuals, leading to the "inflammatory/cytokine hypothesis of depression" [4]. Also, a randomized controlled trial revealed that TNF- $\alpha$ inhibitors (TNFi) can improve depressive symptoms in patients with treatment-resistant depression and increased inflammatory markers [5].

From a clinical perspective, it is of importance to understand whether treatment with TNFi can reduce depressive symptoms in patients with AS. From a pathophysiological point of view, it might be interesting to understand if such an effect would be the result of a reduction of AS-related symptoms, inflammation, or both. Previously, several studies have reported improvements in depressive symptoms in patients with AS after treatment with infliximab [6-8]. Interestingly, observations on the mechanism behind this (potential) effect of infliximab were not equivocal, as correlations of depressive symptoms with either AS-related symptoms (self-reported disease activity) [8] or inflammatory biomarkers [6] have been reported. None of these studies was placebo-controlled or blinded, however, and the observed improvements could have been the result of regression to the mean. In two randomized controlled trials (RCTs), etanercept improved depressive symptoms in patients with AS and non-radiographic axial spondyloarthritis (nr-axSpA), although reported effects were small, and the nature of the effect of TNFi on depressive symptoms was not further explored $[9,10]$.

The objectives of the present study were to evaluate in patients with AS the effect of infliximab on depressive symptoms compared to placebo and to explore the association between depressive symptoms and AS-related symptoms (experienced disease activity and physical functioning) and inflammation over time.

\section{Patients and methods}

Data were retrieved from a sub-study of the Ankylosing Spondylitis Study for the Evaluation of Recombinant Infliximab Therapy (ASSERT), which originally randomized 279 patients. Patients from ASSERT that had been included in the Maastricht University Medical Centre were considered eligible for the current sub-study, and asked to complete a questionnaire on depressive symptoms in parallel to the protocol-required assessments of ASSERT. The ancillary study population consisted of 23 patients, 16 patients randomly assigned to infliximab and 7 to placebo.

\section{Study design}

The design of the ASSERT RCT has been previously reported [11]. Briefly, patients were included in ASSERT if they were 18 years or older and classified as AS according to the modified New York criteria. Patients had to have a Bath AS Disease activity index (BASDAI) score $\geq 4$ and a spinal pain assessment score $\geq 4$ on a visual analog scale [12]. Patients were randomly assigned (3:8 ratio) to receive infusions of $5 \mathrm{mg} / \mathrm{kg}$ infliximab or placebo at weeks $0,2,6,12$, and 18. From week 24 until week 54, all patients received infliximab therapy. The study protocol was reviewed and approved by the independent ethics committee (METC azM/UM). All patients provided written informed consent.

\section{Study outcomes}

Depressive symptoms were assessed with the Center for Epidemiologic Studies Depression scale (CES-D) $[13,14]$. This validated instrument was chosen since it contains fewer somatic items than other instruments [15]. The CES-D consists of 20 items on 
perceived mood and level of functioning during the past week. Every item is scored on a 4-point scale, where $0=$ rarely or none of the time, $1=$ some or litthe of the time, 2 =occasionally or a moderate amount of time, and $3=$ most of the time. The total CES-D score is the sum of all items (range 0 [best] to 60 [worst]). In addition, four CES-D subscales have been defined ("Somatic-retarded activity" [range 0-21], "Depressed affect" [range 0-15], "Positive affect" [range 0-12] and "Interpersonal affect" [range 0-6]), reflecting combinations of varying individual items [16]. A total CES-D score of $\geq 16$ is employed as a cutoff suggestive for clinical depression (i.e., "possible depression") and would warrant a referral for a diagnostic evaluation [14]. Self-reported disease activity and physical function were measured with the BASDAI and Bath AS Functional Index (BASFI), respectively [17]. Inflammation was assessed with the serum CRP. Study outcomes were assessed at weeks 0, 6, 12, 24 , and 54. Both patients and assessors were blinded until week 24 . The main interest of the study was the (between-group difference in) change from baseline CES-D at week 24, in line with ASSERT, in which the main outcome was assessed at the same point in follow-up.

\section{Statistical analysis}

Differences in baseline characteristics between groups were explored with independent $t$ test, Mann Whitney test, or chi-square test, depending on level of measurement and distribution. Fisher's exact test was preferred over chisquare test for small samples (expected count $<5$ ). Chisquare tests (or Fisher's exact tests) and Mann-Whitney $U$ tests were used to respectively compare the proportion of patients with a CES-D score $\geq 16$ and the mean CES-D scores between groups at the different time points.

The course of CES-D scores between groups over time (until week 24, as thereafter both groups were on infliximab) was compared using generalized estimating equation (GEE) analyses. GEE can take into account the within-subject correlation in a longitudinal study, i.e. the dependency that exists between assessments within the same subject $[18,19]$. GEE is focused on estimating the average outcome in the population (population-averaged model), and the model estimates reflect both withinsubject and between-subject effects. It requires an a priori defined "working correlation structure". For this analysis, an "exchangeable" correlation structure was chosen, based on the similar correlations of CES-D scores between time points [19].

First, separate GEE analyses were carried out with either continuous CES-D scores, or dichotomized CES-D score (normal $[<16]$ vs. increased $[\geq 16]$ ), as the outcome (dependent variable). Considering the small sample size, only a limited number of variables could be included in the GEE. Group (infliximab versus placebo) and time (categorical) were included as independent variables, as was as an interaction between group and time (group*time), to test whether there was a difference in the outcome (change from baseline CES-D [continuous] or change from baseline odds of increased CES-D [dichotomized]) between the groups after the first 24 weeks. The GEE analyses were adjusted for baseline CES-D. Second, as we were specifically interested in the mechanism behind (improvement of) depressive symptoms in AS, we explored whether a potential association between infliximab and reduced depressive symptoms remained after adjustment for time-varying AS-specific variables of disease symptoms (BASDAI or BASFI; added as independent variables to the initial model with group, time, group*time and baseline CES-D as independent variables), as well as inflammation (CRP; added as independent variable to the initial model with group, time, group*time and baseline CES-D as independent variables), or both. Of note, BASDAI and BASFI were added in separate models due to collinearity. In ASSERT, treatment allocation was stratified by CRP (within or above 3 times the upper limit of normal); for the current sub-study, we did not adjust for this stratification, as CRP was one of our variables of interest and in light of the sample size. For all analyses, $p<0.05$ was considered statistically significant. Analyses were performed with $R$, version 3.5.3 [20].

\section{Results}

At baseline, the groups were largely comparable in terms of demographics and disease characteristics, although the placebo group contained only male patients and had slightly (though statistically non-significant) higher mean BASFI and Patient Global (Table 1). At weeks 6, 12, 24 and 54, a CES-D score was missing for 1, 2, 0 and 2 patients, respectively; none of the patients had more than 1 missing CES-D score. The mean CES-D score at baseline was 15.5 (SD 9.3) in the infliximab group and 17.3 (SD 5.7) in the placebo group. Fifty-six percent (9 of 16) of the patients in the infliximab group and $43 \%$ (3 of 7) in the placebo-group had a CES-D score $\geq 16$ at baseline, suggestive for clinical depression. The scores on the four CES-D subscales did not differ between groups at baseline (see Additional file 1). When comparing the current sub-study population $(n=23)$ to those who did participate in ASSERT but not part of this sub-study $(n=256)$, the ancillary study patients had slightly higher scores on BASDAI (7.0 [SD 1.1] versus 6.4 [SD 1.6]) and BASFI (6.6 [SD 1.5] versus 5.7 [SD2.0]) (see Additional file 2).

\section{Course of CES-D over time}

In the first 24 weeks after baseline, CES-D in the infliximab group decreased substantially compared to the 
Table 1 Baseline characteristics separately for patients in the infliximab and placebo arm

\begin{tabular}{|c|c|c|c|}
\hline & Infliximab $(\boldsymbol{n}=16)$ & Placebo $(\boldsymbol{n}=7)$ & $p$ \\
\hline Male gender, $n(\%)$ & $11(68.8)$ & $7(100.0)$ & 0.27 \\
\hline Age, years & $38.6(11.6)$ & $44.9(5.8)$ & 0.19 \\
\hline Disease duration, years & $8.3(8.2)$ & $11.5(7.4)$ & 0.37 \\
\hline HLA-B27 positive, $n(\%)$ & $14(87.5)$ & $5(71.4)$ & 0.56 \\
\hline History of uveitis, $n(\%)$ & $6(37.5)$ & $1(14.3)$ & 0.37 \\
\hline History of psoriasis, $n$ (\%) & $0(0.0)$ & $0(0.0)$ & - \\
\hline History of IBD, $n(\%)$ & $2(12.5)$ & $1(14.3)$ & 1.00 \\
\hline BASDAI score, 0-10 & $7.0(1.3)$ & $7.1(0.7)$ & 0.55 \\
\hline BASFI score, 0-10 & $6.3(1.5)$ & $7.2(1.3)$ & 0.21 \\
\hline Patient's global assessment, 0-10 VAS & $6.8(1.4)$ & $7.8(1.2)$ & 0.15 \\
\hline Mander enthesis index, 0-90 & $7.7(8.6)$ & $12.8(14.6)$ & 0.69 \\
\hline Swollen joint index, 0-44 & $2.9(3.7)$ & $3.0(3.9)$ & 0.86 \\
\hline Chest expansion, $\mathrm{cm}$ & $2.0(0.9)$ & $2.5(2.0)$ & 0.87 \\
\hline Night pain, 0-10 VAS & $6.4(2.0)$ & $7.4(1.0)$ & 0.11 \\
\hline CRP level, mg/L & $26.0(24.4)$ & $15.4(16.0)$ & 0.22 \\
\hline Increased CRP, $n(\%)^{*}$ & $13(81.3)$ & $6(85.7)$ & 1.00 \\
\hline CES-D score, 0-60 & $15.5(9.3)$ & $17.3(5.7)$ & 0.66 \\
\hline Increased CES-D, n (\%) ${ }^{\dagger}$ & $9(56.3)$ & $3(42.9)$ & 0.67 \\
\hline
\end{tabular}

Values expressed as mean (SD), unless otherwise indicated

HLA-B27 human leucocyte antigen-B27, IBD inflammatory bowel disease, BASDAI Bath Ankylosing Spondylitis Disease Activity Index, BASFI Bath Ankylosing

Spondylitis Functional Index, VAS visual analog scale, CRP C-reactive protein, CES-D Center for Epidemiologic Studies Depression Scale

*Defined as CRP $>5 \mathrm{mg} / \mathrm{L}$

${ }^{\dagger}$ Defined as a score $\geq 16$, indicating possible depression

placebo group (Fig. 1a). Within groups, the mean (SD) [change from baseline $(\Delta)$, number of patients who completed the CES-D at that time point] CES-D score at week 6 had decreased to 10.3 (SD 7.7) $[\Delta-5.2, n=16]$ in the infliximab group and 15.9 (SD 6.0) $[\Delta-2.5, n=6]$ in the placebo-group ( $p=0.03$ for comparison of absolute CES-D). At 24 weeks, mean CES-D scores were 9.5 (SD 11.4) $[\Delta-6.0, n=16]$ in the infliximab group and 18.0 (SD 6.9) $[\Delta+0.6, n=7]$ in the placebo group $(p=$ 0.02 for comparison of absolute CES-D). At week 54, 30 weeks after the original placebo group had switched to infliximab, the mean CES-D score in the original placebo group had decreased to the same degree as the infliximab group (9.5 (SD 13.1) $[\Delta-9.3, n=5]$ vs. 8.6 (SD 8.4) [ $\Delta-6.9, n=16], p=0.90$ for comparison of absolute CES-D). Similar changes over time were observed in both groups for BASDAI (Fig. 1b).

Exploration of the CES-D subscales showed scores in the infliximab group had improved strongest in the first 24 weeks after baseline for the subscale "Somatic-retarded activity" and to a lesser extent for the subscale "Depressed affect", when compared to the placebo group (see Additional file 1).

At week 6, 25\% (4 of 16) of the infliximab group had a CES-D score $>16$ suggestive for clinical depression, compared to $50 \%$ (3 of 6$)$ in the placebo group $(p=0.33)$
(Fig. 2). After 24 weeks, these proportions were $13 \%$ (2 of 16$)$ and $71 \%$ (5 of 7), respectively $(p=0.01)$. At week 54 , when all patients received infliximab, 20-25\% ( 1 of 5 of the original placebo group, 4 of 16 of the original infliximab group) had a CES-D score $\geq 16$.

\section{GEE analysis of factors associated with CES-D over time}

Differences in disease course between treatment groups over time were explored by GEE. As expected, BASDAI scores in the first 24 weeks were significantly lower in the infliximab group compared to the placebo group $\left(B_{\text {group }}\right.$ time $(24)=-2.97,95 \% \mathrm{CI}-4.51$ to $\left.-1.42, p<0.01\right)$. This effect was partially explained by a reduction in inflammation (reflected by CRP) in the infliximab group, as observed in separate models in which CRP was included in addition to treatment (data not shown).

Further exploration of depressive symptoms by GEE revealed a (borderline non-significant) larger improvement in CES-D scores in the infliximab group compared to the placebo group after the first 24 weeks (model 1A: $B_{\text {group"time (24) }}=-6.63,95 \% \mathrm{CI}-13.35$ to $0.09, p=0.05$ ). After adjustment for BASDAI, no association was observed anymore between infliximab and change in CESD scores over time (model 1B: $B_{\text {group"time }(24)}=-2.66$, $95 \% \mathrm{CI}-9.44$ to $4.11, p=0.44$ ), while BASDAI itself was significantly associated with CES-D scores (model 1B: 

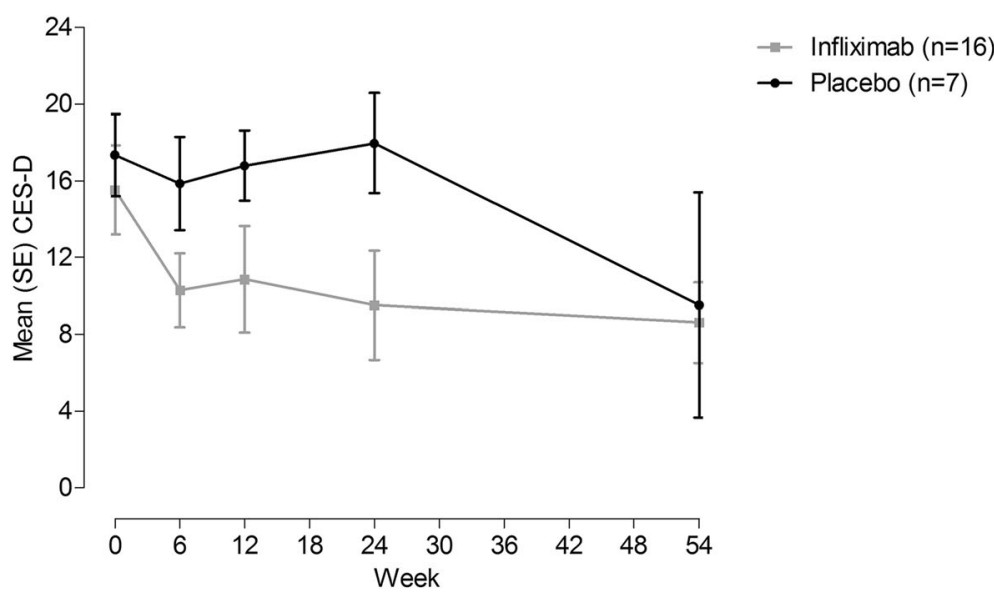

A $\underset{\text { Placebo-controlled }}{\longrightarrow} \underset{\text { Open-label extension }}{\longrightarrow}$

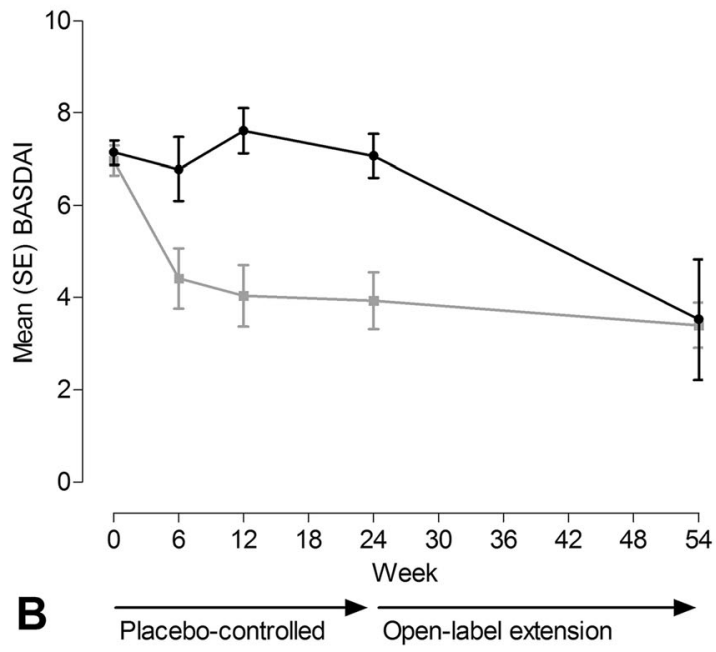

Fig. 1 CES-D (a) and BASDAI (b) scores over time according to treatment group. Figure caption: Course of mean CES-D (a) and BASDAI (b) scores over time, by treatment group. During the open-label extension, all patients received infliximab. BASDAl, Bath Ankylosing Spondylitis Disease Activity Index; CES-D, Center for Epidemiologic Studies Depression Scale; SE, standard error

$B_{\text {BASDAI }}=1.34,95 \%$ CI 0.53 to $2.15, p<0.01$ ) (Table 2). Adjustment for CRP instead of BASDAI had a notably smaller effect on the association between infliximab treatment and CES-D scores (model 1C: $B_{\text {group"time(24) }}=$ $-5.71,95 \% \mathrm{CI}-12.53$ to $1.12, p=0.10)$. Finally, when both BASDAI and CRP were included, BASDAI remained associated with CES-D scores, while CRP was not (model 1D, Table 2).

When exploring the odds of possible depression (CES$D$ score $\geq 16$ ) as the outcome, while adjusting for baseline CES-D status (increased $(>16)$ vs normal $(<16)$ ), findings were similar: inflixima $\bar{b}$ treatment was associated with a larger reduction in odds of having an increased CES-D after 24 weeks (model 2A: $\mathrm{OR}_{\text {group }}$ time(24) $=0.02,95 \% \mathrm{CI} 0.00$ to $0.72, p=0.03$ ), but after adjustment for BASDAI, this association was no longer significant (model 2B: $\mathrm{OR}_{\text {group }}=0.03$, 95\%CI 0.00 to $1.32, p=0.07$ ) (Table 2). After adjustment for CRP instead of BASDAI, a similar observation was made (model 2C: OR $_{\text {group }}$ time(24) $=0.03,95 \% \mathrm{CI} 0.00$ to 1.30 , $p=0.07$ ) (Table 2). Adjustment for both BASDAI and CRP resulted in none of these (treatment or BASDAI or CRP) being significantly associated with increased CESD scores (model 2D, Table 2).

Analyses using BASFI instead of BASDAI led to similar results: after adjustment for BASFI, the initial association between treatment group and CES-D scores was no longer observed, and when both BASFI and CRP were included, BASFI was associated with CES-D, while CRP was not (models 3A-3D, Table 3). Using the odds of possible depression (CES-D score $\geq 16$ ) as outcome yielded similar results, although BASFI remained 


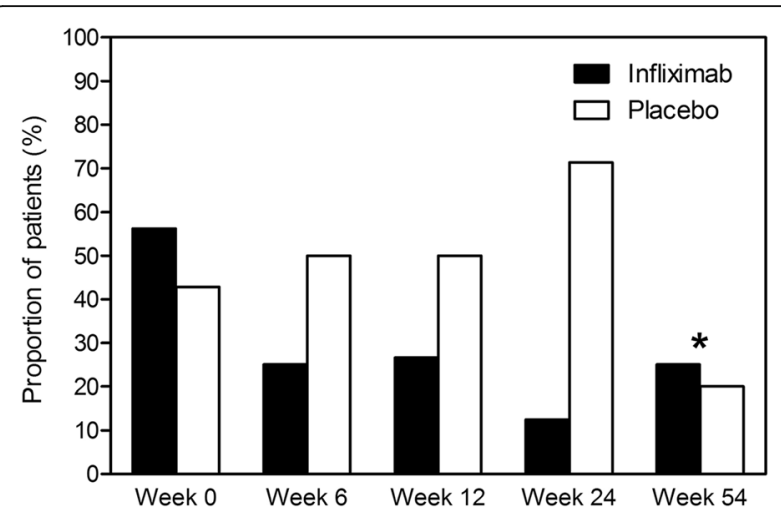

Fig. 2 Proportion of patients with CES-D score $\geq 16$ over time according to treatment group. Figure caption: Proportion of patients with an increased CES-D score $(\geq 16)$ at each assessment, by treatment group. *From week 24 onwards, all patients received infliximab. CES-D, Center for Epidemiologic Studies Depression Scale

associated with possible depression, also after adjustment for CRP (models 4A-4D, Table 3).

\section{Discussion}

This study showed that depressive symptoms are common in patients with AS that have high disease activity. Infliximab improved depressive symptoms in patients with AS after 24 weeks of treatment. This effect could largely be explained by the effect of infliximab on selfreported symptoms of AS.

Over half of the patients with AS and active disease had a CES-D score $\geq 16$ at baseline, indicative for possible depression. This high proportion seems to be on the upper end of the prevalence range as reported in the literature, but is likely the result of the inclusion criteria for ASSE RT, which required patients to have active disease [1]. For comparison, in control populations in the Netherlands, a possible depression as measured by increased CES-D score has been reported in 5-22\% [21-23].

The reduction in depressive symptoms in the infliximab group occurred already within the first weeks and was maintained during the remainder of follow-up. Importantly, treatment with infliximab was not only associated with a decrease in depressive symptoms, but also with decreased odds of CES-D scores above the threshold for (probable) depression. The substantial reduction (an estimated 98\% reduction in odds of having an increased CES-D after 24 weeks of weeks of infliximab compared to placebo), although certainly an overestimation of the effect of infliximab on true clinical depression, seems clinically relevant. This implicates that, at least in a population of patients with active AS, infliximab not only decreases the severity of depressive symptoms and odds of probable depression, but potentially even lowers the odds of true clinical depression. Of note, even after up to 54 weeks of treatment, $20-25 \%$ of both groups still had CES-D scores suggestive of possible depression.

Comparing our results with other studies that investigated the effect of TNFi on depressive symptoms in AS is difficult, because other study designs and/or instruments were used to assess depressive symptoms (in none the CES-D was used) [6-10]. Two RCTs assessed the effect of etanercept on depressive symptoms as a secondary outcome or in post hoc analysis. The first study, comparing sulfasalazine to etanercept among 566 patients with AS, reported significant improvements in depressive symptoms after 16 weeks in both treatment arms, with larger improvements in the etanercept group [9]. In the second study, where 215 patients with nraxSpA were treated with etanercept or placebo for 12 weeks followed by etanercept for all subjects for another 12 weeks, depressive symptoms did not differ between groups after 12 weeks or 24 weeks, but had improved from baseline after 24 weeks in both groups [10]. In addition, three observational studies on the effect of infliximab on depressive symptoms in AS were published, with a total of 52 patients and all of limited duration (6-12 weeks) [6-8]. In line with our findings, in these observational studies, similar proportions of patients with depression scores above the threshold for the respective depression instrument used at baseline and over time were observed $[7,8]$. Also, improvements in depressive symptoms occurred already within the first weeks after initiation of infliximab. Of note, none of these studies explored whether these improvements can be fully explained by improvements in symptoms or inflammation.

It remains challenging whether our results can help to understand if AS has a direct (inflammatory) or indirect (through pain and limitations) effect on depressive symptoms. The association between treatment with infliximab and improvement of depressive symptoms largely disappeared after adjustment for AS-specific symptoms, i.e. self-reported disease activity and physical function, suggesting the change in depressive symptoms in these patients is (at least partially) secondary to changes in their AS-related symptoms. Of note, the relationship between self-reported depressive symptoms and self-reported AS-related symptoms could (partially) be bidirectional. In rheumatoid arthritis, for example, the patient visual analog scale component of the Disease Activity Score (DAS28) has been strongly associated with depression [24]. Likewise, in the current study, responses on BASDAI or BASFI could be influenced by the patient's emotional state. Additionally, it is known some covariance exists between all self-reported measures, which likely adds to some overestimation of the observed associations. Notwithstanding, when considering an alternative explanation for the improvements in 


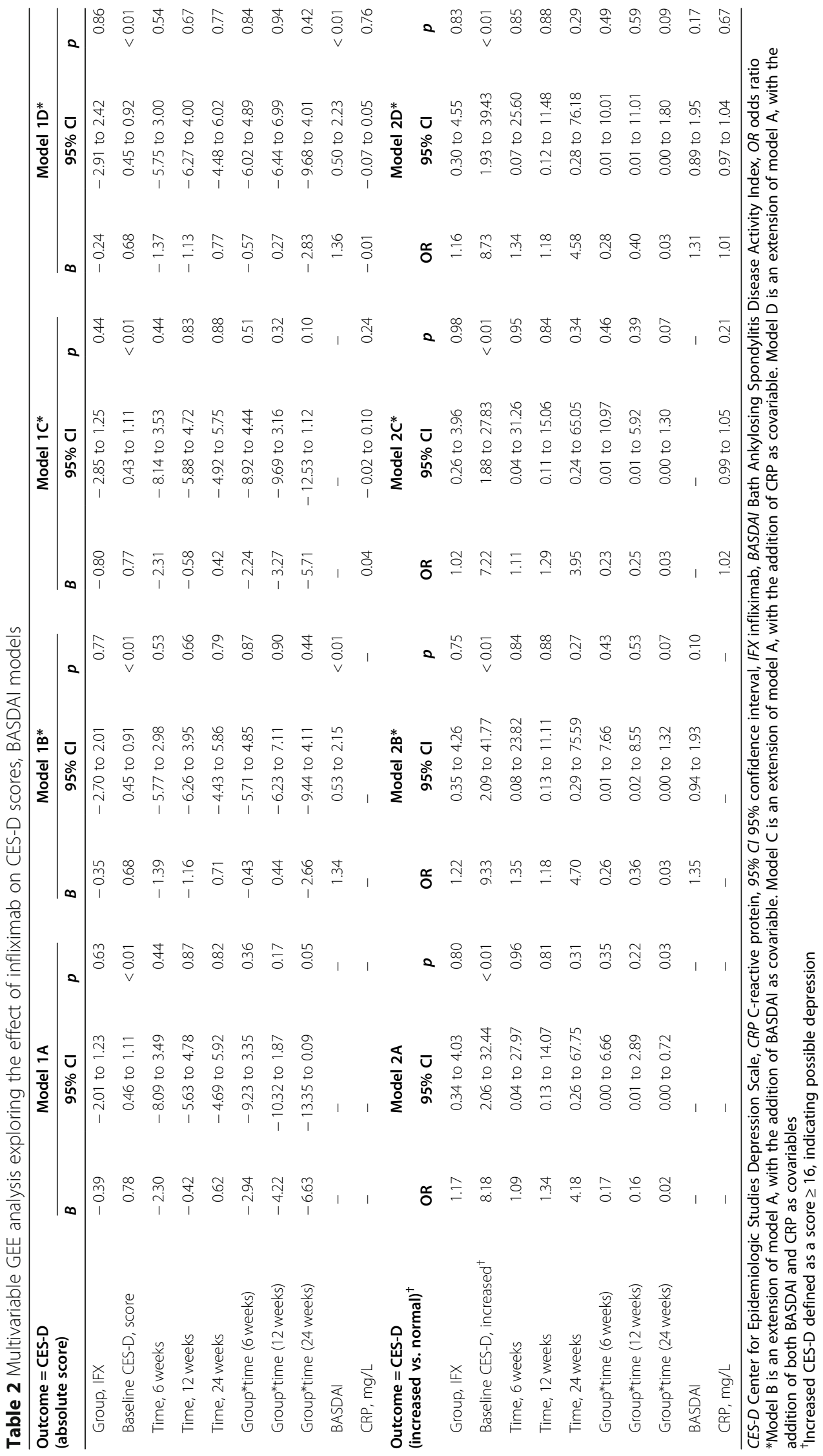




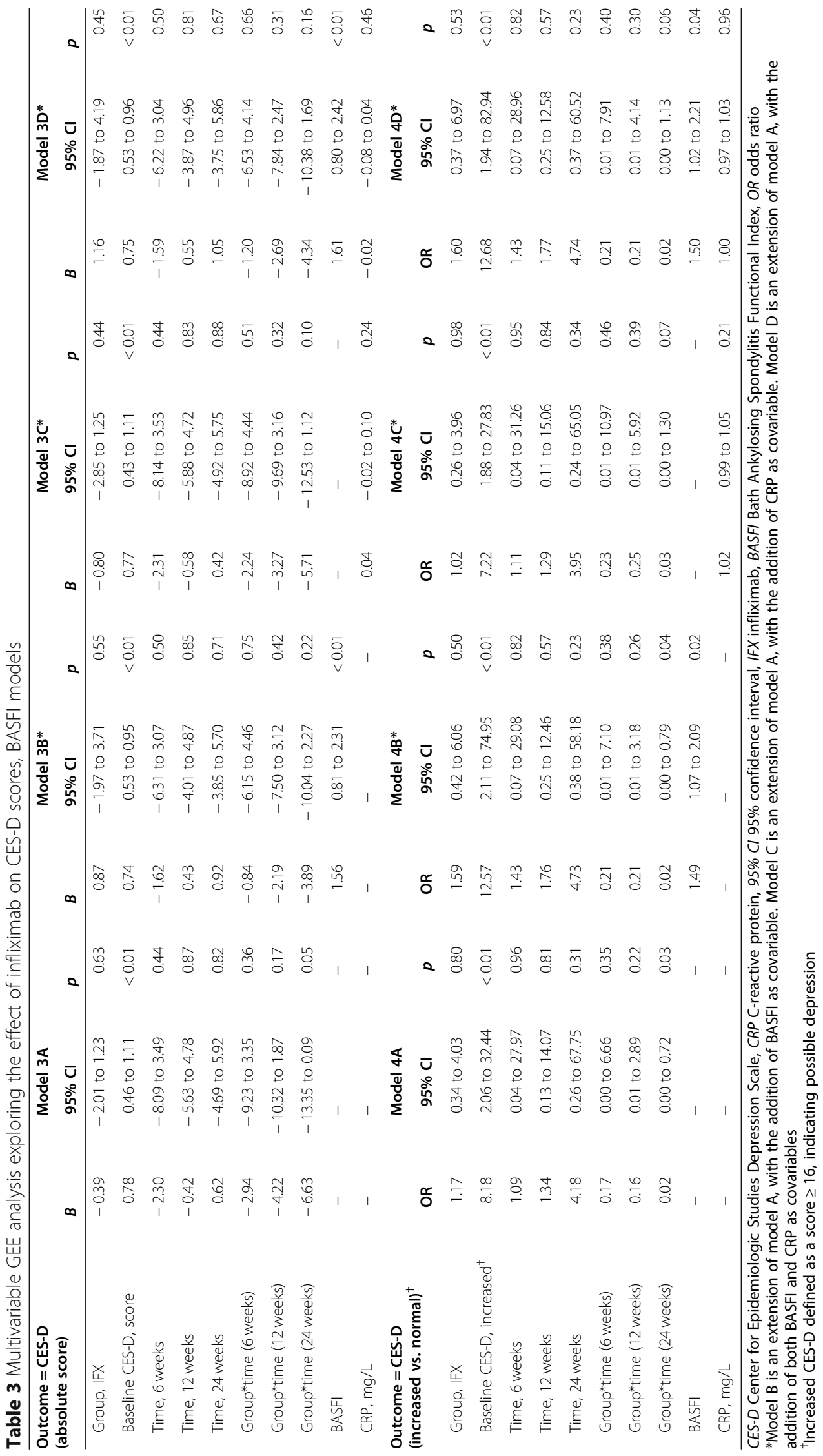


depressive symptoms, i.e. a reduction in inflammation, CRP did not seem to independently/directly contribute to the effect of infliximab treatment on depressive symptoms: adjustment for CRP did have a minor impact on the association between infliximab and depressive symptoms when compared to the effect of BASDAI or BASFI, suggesting little mediation of the effect of infliximab via CRP-mediated inflammation. This further suggests the mechanism behind depressive symptoms in these patients is mainly based on the impact of AS-related symptoms. It should be noted, however, that due to the small sample size, these secondary analyses were only exploratory and no elaborate path analyses could be conducted. Nonetheless, the association between CRP and depressive symptoms, compared with BASDAI or BASFI, was not only statistically non-significant but also very small numerically, suggesting a direct effect of CRP-mediated inflammation would be little (if any) in a larger sample. Overall, it remains challenging to unravel the intricate relationships between the inflammatory pathophysiological process behind AS, AS-related symptoms, and depression. While CRP is commonly used as inflammatory biomarker in axSpA, we cannot rule out CRP is not appropriate as a biomarker to identify a potential link between inflammation and depression in this disease. While the current results do not allow us to draw firm conclusions regarding these associations between markers of inflammation and depression, our data suggests other inflammatory biomarkers are likely more interesting to further explore than CRP.

In addition, two other interesting observations can be made. First, while all patients had high BASDAI and BASFI scores at baseline, only half of these patients had CES-D scores above the threshold for possible depression, indicating the relation between experienced pain or functional limitations and depression is not an absolute one. Second, baseline CES-D remained strongly associated with the course of CES-D over time, even when accounting for BASDAI or BASFI. This suggests that, while the effect of infliximab on depressive symptoms seems to be mostly mediated by improvement in pain and functional limitations, depressive symptoms as measured with the CES-D are additional and distinct phenomena rather than only a reflection of these pain and limitations. It would be interesting to further explore which patients with active disease have an increased susceptibility to depression, for example as a result of genetic predisposition or personality and coping traits [25]. On this line, it has recently been shown that illness perceptions have an important role in the relationship between back pain and mental health outcomes [26]. Further, in axSpA, patients with comorbid depression are much more likely to suffer from other mental health and substance abuse disorders, also suggesting an underlying vulnerability [27].

The main limitation of this study is the small number of patients restricting the power to detect significant changes and limiting the number of covariables that could be included in the models. However, strong and clinically relevant absolute changes and improvements in depressive symptoms were observed. Further, we should realize that the CES-D is a screening questionnaire which cannot be used to diagnose depression, for which the gold standard still is psychiatric interviewing and examination. On this line it should be noted the questions in the CES-D refer to the past week, which might further reflect reactive depressive symptoms (instead of clinical depression/chronic depression), overestimating the proportion with actual depression. The associations as observed in the analyses of the current study are possibly an overestimation (numerically), and the effect of infliximab on true (physician-diagnosed) clinical depression might be smaller in daily practice. Finally, we did not have data available on the individual BASDAI items, precluding analysis of the contribution of each item.

The findings in the present study have several implications. Rheumatologists should be aware of the high prevalence of depressive symptoms in patients with AS and active disease, while considering that these symptoms are not strictly a result of pain and loss of functioning. In addition, our results suggest that treatment with a TNFi is beneficial for depressive symptoms in the majority of this population. Still, a proportion of patients seem to maintain clinically significant depressive symptoms despite TNFi treatment, which might require specialized treatment.

\section{Conclusions}

The prevalence of depressive symptoms was high among this patient population with active AS. TNFi treatment improved the depressive symptoms of AS patients; our data suggest that this benefit seems an indirect effect of TNFi treatment on AS-related symptoms. Appropriate management of depression in AS deserves attention in clinical practice.

\section{Supplementary information}

Supplementary information accompanies this paper at https://doi.org/10. 1186/s13075-020-02305-w.

Additional file 1. CES-D subscale scores over time. Table and graph with CES-D subscale scores over time, by group.

Additional file 2. Comparison of ASSERT subjects who did or did not participate in the ancillary depression study. Table with patient characteristics of ASSERT subjects who did or did not participate in the ancillary depression study. 


\section{Abbreviations}

AS: Ankylosing spondylitis; ASSERT: Ankylosing Spondylitis Study for the Evaluation of Recombinant Infliximab Therapy; BASDAl: Bath Ankylosing Spondylitis Disease Activity Index; BASFl: Bath Ankylosing Spondylitis Functional Index; CES-D: Center for Epidemiologic Studies Depression scale; CRP: C-reactive protein; GEE: Generalized estimating equation; $\mathrm{nr}$ axSpA: Non-radiographic axial spondyloarthritis; RCT: Randomized controlled trial; TNF: Tumor necrosis factor; TNFi: Tumor necrosis factor alpha inhibitor

\section{Acknowledgements}

Not applicable.

\section{Authors' contributions}

$A B$ and DvdH designed the study. CS, AvT, TS, DvdH, RL, and AB were involved in data acquisition and/or data management. CW, CS, OS, AvT, and $A B$ analyzed the data. CW, CS, AvT, RL, and AB critically interpreted the results. $C W, C S, A v T$, and $A B$ were involved in drafting the manuscript. All authors revised the manuscript critically for important intellectual content and approved the final manuscript.

\section{Funding}

This sub-study of the ASSERT trial is performed without any financial support. ASSERT was sponsored by Janssen Biologics. Janssen Biologics had no role in the study design or in the collection, analysis, or interpretation of the data; the writing of the manuscript; or the decision to submit the manuscript for publication.

\section{Availability of data and materials}

All data generated or analyzed during this study are included in this published article and its supplementary information files.

\section{Ethics approval and consent to participate}

The ethics committee from the Maastricht University Medical Centre approved this substudy. All patients provided written informed consent.

\section{Consent for publication}

Not applicable.

\section{Competing interests}

The authors declare that they have no competing interests.

\section{Author details}

'Department of Internal Medicine, Division of Rheumatology, Maastricht University Medical Centre, PO Box 5800, Maastricht 6202 AZ, The Netherlands. ${ }^{2}$ Care and Public Health Research Institute (CAPHRI), Maastricht University, Maastricht, the Netherlands. ${ }^{3}$ Department of Internal Medicine, Erasmus Medical Centre, Rotterdam, the Netherlands. ${ }^{4}$ Department of Psychiatry and Neuropsychology, School for Mental Health and Neuroscience (MHeNs), Maastricht University, Maastricht, the Netherlands. ${ }^{5}$ Department of Clinical Immunology \& Rheumatology, Amsterdam Rheumatology Centre, University of Amsterdam, Amsterdam, the Netherlands. ${ }^{6}$ Department of Rheumatology, Zuyderland Medical Centre, Heerlen, the Netherlands. ${ }^{7}$ Department of Rheumatology, Leiden University Medical Centre, Leiden, the Netherlands.

Received: 17 March 2020 Accepted: 25 August 2020

Published online: 29 September 2020

\section{References}

1. Zhao S, Thong D, Miller N, et al. The prevalence of depression in axial spondyloarthritis and its association with disease activity: a systematic review and meta-analysis. Arthritis Res Ther. 2018;20:140.

2. Lowe B, Willand L, Eich W, et al. Psychiatric comorbidity and work disability in patients with inflammatory rheumatic diseases. Psychosom Med. 2004;66: 395-402.

3. Martindale J, Smith J, Sutton CJ, et al. Disease and psychological status in ankylosing spondylitis. Rheumatology (Oxford). 2006;45:1288-93.

4. Miller $\mathrm{AH}$, Raison $\mathrm{CL}$. The role of inflammation in depression: from evolutionary imperative to modern treatment target. Nat Rev Immunol. 2016;16:22-34.
5. Raison $\mathrm{CL}$, Rutherford $\mathrm{RE}$, Woolwine $\mathrm{BJ}$, et al. A randomized controlled trial of the tumor necrosis factor antagonist infliximab for treatment-resistant depression: the role of baseline inflammatory biomarkers. JAMA Psychiatry. 2013;70:31-41.

6. Arisoy O, Bes C, Cifci C, et al. The effect of TNF-alpha blockers on psychometric measures in ankylosing spondylitis patients: a preliminary observation. Rheumatol Int. 2013;33:1855-64.

7. Ertenli I, Ozer S, Kiraz S, et al. Infliximab, a TNF-alpha antagonist treatment in patients with ankylosing spondylitis: the impact on depression, anxiety and quality of life level. Rheumatol Int. 2012;32:323-30.

8. Ersozlu-Bozkirli ED, Keskek SO, Bozkirli E, et al. The effect of infliximab on depressive symptoms in patients with ankylosing spondylitis. Acta Reumatol Port. 2015;40:262-7.

9. van der Heijde D, Braun J, Dougados M, et al. Sensitivity and discriminatory ability of the Ankylosing Spondylitis Disease Activity Score in patients treated with etanercept or sulphasalazine in the ASCEND trial. Rheumatology (Oxford). 2012;51:1894-905.

10. Dougados M, Tsai WC, Saaibi DL, et al. Evaluation of health outcomes with etanercept treatment in patients with early nonradiographic axial spondyloarthritis. J Rheumatol. 2015;42:1835-41.

11. van der Heijde D, Dijkmans B, Geusens P, et al. Efficacy and safety of infliximab in patients with ankylosing spondylitis: results of a randomized, placebo-controlled trial (ASSERT). Arthritis Rheum. 2005;52:582-91.

12. Garrett $S$, Jenkinson T, Kennedy LG, et al. A new approach to defining disease status in ankylosing spondylitis: the Bath Ankylosing Spondylitis Disease Activity Index. J Rheumatol. 1994;21:2286-91.

13. Radloff LS. The CES-D Scale: a self-report depression scale for research in the general population. Appl Psychol Meas. 1977;1:385-401.

14. Smarr KL, Keefer AL. Measures of depression and depressive symptoms: Beck Depression Inventory-II (BDI-II), Center for Epidemiologic Studies Depression Scale (CES-D), Geriatric Depression Scale (GDS), Hospital Anxiety and Depression Scale (HADS), and Patient Health Questionnaire-9 (PHQ-9). Arthritis Care Res (Hoboken). 2011;63(Suppl 11):S454-66.

15. Blalock SJ, DeVellis RF, Brown GK, et al. Validity of the Center for Epidemiological Studies Depression Scale in arthritis populations. Arthritis Rheum. 1989:32:991-7.

16. Ensel WM. 4 - Measuring Depression: The CES-D Scale. In: Lin N, Dean A, Ensel WM. Social Support, Life Events, and Depression. Orlando: Academic Press; 1986. p. 51-70.

17. Calin A, Garrett S, Whitelock H, et al. A new approach to defining functional ability in ankylosing spondylitis: the development of the Bath Ankylosing Spondylitis Functional Index. J Rheumatol. 1994;21:2281-5.

18. Zeger SL, Liang KY, Albert PS. Models for longitudinal data: a generalized estimating equation approach. Biometrics. 1988;44:1049-60.

19. Twisk JW. Longitudinal data analysis. A comparison between generalized estimating equations and random coefficient analysis. Eur J Epidemiol. 2004; 19:769-76.

20. R Core Team. R: A language and environment for statistical computing. Vienna: R Foundation for Statistical Computing; 2019. URL: http://www.R-project.org.

21. Bouma J, Ranchor A, Sanderman R, et al. Het meten van symptomen bij depressie met de CES-D, een handleiding [Measurement of depressive symptoms with the CES-D, a manual]. Groningen, Noordelijk Centrum voor Gezondheidsvraagstukken, Rijksuniversiteit Groningen 19952-24.

22. Pouwer F, Beekman AT, Nijpels G, et al. Rates and risks for co-morbid depression in patients with type 2 diabetes mellitus: results from a community-based study. Diabetologia. 2003;46:892-8.

23. Schroevers MJ, Sanderman R, van Sonderen E, et al. The evaluation of the Center for Epidemiologic Studies Depression (CES-D) scale: depressed and positive affect in cancer patients and healthy reference subjects. Qual Life Res. 2000;9:1015-29.

24. Cordingley L, Prajapati R, Plant D, et al. Impact of psychological factors on subjective disease activity assessments in patients with severe rheumatoid arthritis. Arthritis Care Res (Hoboken). 2014;66:861-8.

25. Webers C, Vanhoof L, Leue C, et al. Depression in ankylosing spondylitis and the role of disease-related and contextual factors: a cross-sectional study. Arthritis Res Ther. 2019;21:215.

26. van Lunteren M, Scharloo M, Ez-Zaitouni Z, et al. The impact of illness perceptions and coping on the association between back pain and health outcomes in patients suspected of having axial spondyloarthritis: data from the SPondyloArthritis Caught Early Cohort. Arthritis Care Res (Hoboken). 2018;70:1829-39. 
27. Zhao SS, Solomon DH, Goodson NJ. Comment on: comorbidity burden in axial spondyloarthritis: a cluster analysis: reply. Rheumatology (Oxford). 2020; 59:692-3.

\section{Publisher's Note}

Springer Nature remains neutral with regard to jurisdictional claims in published maps and institutional affiliations.

Ready to submit your research? Choose BMC and benefit from:

- fast, convenient online submission

- thorough peer review by experienced researchers in your field

- rapid publication on acceptance

- support for research data, including large and complex data types

- gold Open Access which fosters wider collaboration and increased citations

- maximum visibility for your research: over $100 \mathrm{M}$ website views per year

At $\mathrm{BMC}$, research is always in progress. 